\title{
PHOTOMETRIC STEREO SHAPE-AND-ALBEDO-FROM-SHADING FOR PIXEL- LEVEL RESOLUTION LUNAR SURFACE RECONSTRUCTION
}

\author{
Wai Chung Liu, Bo Wu* \\ Dept. of Land Surveying and Geo-Informatics, Faculty of Construction and Environment, The Hong Kong Polytechnic University, \\ Hung Hom, Hong Kong - mo.liu@ connect.polyu.hk; bo.wu@ polyu.edu.hk
}

\author{
Commission III, ICWG III/II
}

KEY WORDS: Moon, DEM, Shape and Albedo from Shading, Photometric Stereo, LROC NAC

\begin{abstract}
:
Shape and Albedo from Shading (SAfS) techniques recover pixel-wise surface details based on the relationship between terrain slopes, illumination and imaging geometry, and the energy response (i.e., image intensity) captured by the sensing system. Multiple images with different illumination geometries (i.e., photometric stereo) can provide better SAfS surface reconstruction due to the increase in observations. Photometric stereo SAfS is suitable for detailed surface reconstruction of the Moon and other extraterrestrial bodies due to the availability of photometric stereo and the less complex surface reflecting properties (i.e., albedo) of the target bodies as compared to the Earth. Considering only one photometric stereo pair (i.e., two images), pixel-variant albedo is still a major obstacle to satisfactory reconstruction and it needs to be regulated by the SAfS algorithm. The illumination directional difference between the two images also becomes an important factor affecting the reconstruction quality. This paper presents a photometric stereo SAfS algorithm for pixel-level resolution lunar surface reconstruction. The algorithm includes a hierarchical optimization architecture for handling pixel-variant albedo and improving performance. With the use of Lunar Reconnaissance Orbiter Camera - Narrow Angle Camera (LROC NAC) photometric stereo images, the reconstructed topography (i.e., the DEM) is compared with the DEM produced independently by photogrammetric methods. This paper also addresses the effect of illumination directional difference in between one photometric stereo pair on the reconstruction quality of the proposed algorithm by both mathematical and experimental analysis. In this case, LROC NAC images under multiple illumination directions are utilized by the proposed algorithm for experimental comparison. The mathematical derivation suggests an illumination azimuthal difference of 90 degrees between two images is recommended to achieve minimal error in SAfS reconstruction while results using real data presents similar pattern. Although the algorithm is designed for lunar surface reconstruction, it is likely to be applicable on other extraterrestrial bodies such as Mars. The results and findings from this research is of significance for the practical use of photometric stereo and SAfS in the domain of planetary remote sensing and mapping.
\end{abstract}

\section{INTRODUCTION}

Shape and Albedo from Shading (SAfS) is characterized by its ability to reconstruct 3D shapes with subtle details on an image. It is able to produce DEMs and other 3D shapes with resolution comparable to the image(s). SAfS utilizes the relationship between 3D geometry and energy reflection for reconstruction, and therefore it also works on single image shape recovery. The geometric information retrieval from SAfS can be less stable compared to other modelling techniques such as photogrammetry and laser altimetry, this is because SAfS deals with the relative difference between adjacent pixels and extra uncertainties are introduced by the reflecting behaviour of surface material (i.e., reflectance models and albedo). Yet, it is able to produce terrain models where other modelling techniques are not available, and with details better than those techniques (Wu et al., 2017).

SAfS techniques depend largely on the understanding of reflectance models which describe the relationship between surface orientation and energy response. This research has been long studied and various practical models were developed (McEwen, 1991; Hapke, 2002). Horn (1977; 1990) studied and formulated the algorithm for shape recovery from shading information; the use of SfS techniques in lunar mapping was also suggested (Horn, 1990) due to the less complex surface albedo on moon and perhaps other extra-terrestrial bodies. Grumpe et al. (2014) and Wu et al. (2017) combined and regulated SfS or SAfS by low-resolution lunar DEMs obtained from photogrammetry and laser altimetry. This strategy allows generation of pixel-level resolution lunar DEMs with their overall geometry comparable to the low-resolution DEMs.

Photometric stereo refers to imaging the same object under different illumination conditions (Woodham, 1980), shading from various illumination direction can provide extra information about the underlying shape and thus improving the quality of 3D reconstruction. Woodham (1980) developed the basic theory of photometric stereo as a way to obtain surface orientation and verified with synthetic examples. Lohse and Heipke (2004) developed the Multi-Image Shape from Shading (MI-SfS) algorithm for lunar mapping. The core concept of MISfS is similar to photometric stereo and they were able to produce high resolution lunar DEMs where image matching fails due to less distinctive texture.

In photometric stereo the azimuthal differences between illumination directions may affect the quality of reconstruction, this is more apparent when only one photometric stereo pair (i.e., two images) is available. This paper firstly describes an iterative hierarchical SAfS algorithm using photometric stereo, followed by the mathematical derivation of the effect of illumination azimuthal differences on SAfS performance. The derivation is then verified by real data using LROC NAC images and the described SAfS algorithm. Each of the SAfS results only utilizes one pair of photometric stereo (i.e., double image SAfS). The analysis and findings are concluded afterwards. 


\section{PHOTOMETRIC STEREO SAFS}

\subsection{Overview of approach}

Photometric stereo SAfS majorly contains two steps: (1) Gradient estimation from reflectance; and (2) Shape reconstruction from gradients. The first step 'Gradient estimation from reflectance' estimates the surface normal of each pixel base on the image intensity, geometric reflectance function and albedo. The output of this step is a vector field and each vector on the field represents a surface normal. This surface normal field is able to reproduce the captured image through the geometric reflectance function and the albedo. The second step 'Shape reconstruction from gradients' generates the DEM according to the computed surface normal by evaluating the relationship between adjacent height nodes. After this step, the DEM should inherit as much information in the surface normal field as possible and will be able to preserve subtle details on the images.

The algorithm uses a hierarchical optimization strategy by down-sampling the images and an initial DEM (i.e., can be initialized by a flat plane, etc.) before SAfS and increases its resolution by a factor of 2 iteratively until it reaches the desired resolution. The current architecture is also named Cascade Multi-grid (Capanna et al., 2013) which will not revisit and optimize lower resolution components again; however general control throughout all hierarchies is present to regulate optimization. Optimization per hierarchy is iterative and will terminate when stopping criteria is satisfied. The reconstruction adopts the Modified Jacobi relaxation scheme (Horn, 1990) to balance between convergence and data inter-dependencies.

\subsection{Reflectance-based shape reconstruction}

Shape reconstruction from shading information depends on the relationship between the surface normal of the object (i.e., slopes and aspects) and the intensity captured by sensors under certain illumination and imaging geometry. The illumination and imaging conditions are usually known as assumed in this article. At any pixel, the recorded intensity $I$ can be expressed as:

$$
I=A G(p, q)
$$

where $\quad I=$ image intensity

$A=$ albedo

$G(p, q)=$ geometric reflectance function

Albedo $A$ represents the ability of the surface material to scatter or reflect incoming light. The value of albedo lies between zero to unity while it is very unlikely that $(A=0)$. The value of albedo can be obtained empirically or be estimated using certain initial estimates of the underlying shape (e.g., assuming to be a flat plane initially). $G(p, q)$ denotes the geometric reflectance function which relates surface normal $(p, q)$ of a point and its reflectance. Well-known reflectance models include the Lambertian model, the Lunar-Lambertian model (McEwen,1991) and Hapke model (Hapke, 2002). The surface normal $(p, q)$ of a point is described by the partial derivatives (i.e., tangent) of the surface $(Z=S(x, y))$ along $\mathrm{x}$ - and $\mathrm{y}$ direction at that point:

$$
p=\frac{\partial S(x, y)}{\partial x} ; q=\frac{\partial S(x, y)}{\partial y}
$$

Given certain initial estimate of the surface normal $\left[\begin{array}{l}p_{0} \\ q_{0}\end{array}\right]$ and albedo $A$, the observation equation can be setup by linearizing $G(p, q)$ to solve for iterative update of the surface normal:

$$
\frac{\partial G_{0}}{\partial p} \Delta p+\frac{\partial G_{0}}{\partial q} \Delta q=\frac{I}{A}-G_{0}
$$

$$
\text { where } \quad \begin{aligned}
G_{0} & =G\left(p_{0}, q_{0}\right) \\
\Delta p & =\text { update for } p \\
\Delta q & =\text { update for } q
\end{aligned}
$$

With multiple images (i.e., photometric stereo pairs), a set of equation (3), one for each image, can be setup and the optimization can be expressed as minimizing the error term:

$$
\sum_{1}^{k}\left(\left.G_{0}\right|_{k}+\left.\frac{\partial G_{0}}{\partial p}\right|_{k} \Delta p+\left.\frac{\partial G_{0}}{\partial q}\right|_{k} \Delta q-\frac{I_{k}}{A}\right)^{2}
$$

where $(k \geq 2)=$ number of available image

A vector field representing the surface normal at each pixel is produced after the aforementioned optimization. Shape recovery from surface normal requires the surface normal field to be integrable, which is formulated as:

$$
\frac{\partial p}{\partial y}=\frac{\partial q}{\partial x}
$$

This implies that for all possible routes between any two points on a surface, the integrated (i.e., summed) height differences of each route must be identical. Some works enforce such condition directly on the surface normal field (Frankot and Chellappa, 1988; Horn, 1990). This research enforces integrability by directly adjusting the elevation nodes of the underlying DEM such that the DEM best represents the surface normal vector field resulted from equation (4). The pixel-variant albedo can be retrieved from the optimized SAfS DEM and the images.

\section{EFFECT OF ILLUMINATION DIRECTIONAL DIFFERENCE TO PHOTOMETRIC STEREO SAFS}

\subsection{Derivation of Photometric Stereo SAfS solution}

Considering any single point $O$ on a surface, photometric stereo SAfS estimates its surface normal by evaluating the slope of $O$ along each of the available illumination directions. Now consider Figure 1 for the case of only one photometric stereo pair is available (i.e., double image SAfS): centring at point $O$, a unit vector $\left[\begin{array}{l}\widehat{p_{L 1}} \\ q_{L 1}\end{array}\right]$ is pointing towards the illumination direction 1 , the horizontal axis $p$ of the Cartesian coordinate frame is aligned to $\left[\begin{array}{c}\widehat{p_{L 1}} \\ \widehat{q_{L 1}}\end{array}\right]$ so that $\left(\left[\begin{array}{c}\widehat{p_{L 1}} \\ \widehat{q_{L 1}}\end{array}\right]=\left[\begin{array}{l}1 \\ 0\end{array}\right]\right)$. Another unit vector $\left[\begin{array}{l}\widehat{p_{L 2}} \\ \widehat{q_{L 2}}\end{array}\right]$ is pointing towards illumination direction 2 which is $\alpha$ degrees counter-clockwise from illumination direction 1 . The relationship between the two unit vectors can be denoted as:

$$
\left[\begin{array}{l}
\widehat{p_{L 2}} \\
\widehat{q_{L 2}}
\end{array}\right]=R(\alpha)\left[\begin{array}{l}
\widehat{p_{L 1}} \\
\widehat{q_{L 1}}
\end{array}\right]=\left[\begin{array}{cc}
\cos \alpha & -\sin \alpha \\
\sin \alpha & \cos \alpha
\end{array}\right]\left[\begin{array}{l}
1 \\
0
\end{array}\right]=\left[\begin{array}{c}
\cos \alpha \\
\sin \alpha
\end{array}\right]
$$

The term $\alpha$ is the azimuthal difference between the two illumination directions, $\alpha$ is negative when the rotation is in clock-wise direction. It is assumed that the zenith angles of both illumination sources are non-zero (i.e., not parallel to the Z-axis 
in 3D space) so that the illumination directions are non-zero when projected to the $2 \mathrm{D}$ horizontal space.

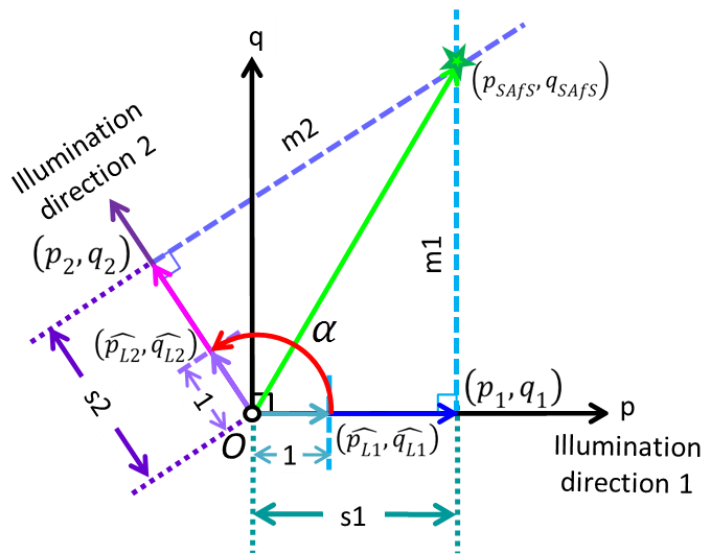

Figure 1. Photometric stereo SAfS at point $O$

The task of SAfS is to estimate the slope at $O$ along the two directions pointed by $\left[\begin{array}{c}\widehat{p_{L 1}} \\ \widehat{q_{L 1}}\end{array}\right]$ and $\left[\begin{array}{l}\widehat{p_{L 2}} \\ \widehat{q_{L 2}}\end{array}\right]$. This can be expressed as solving for two scalars $s_{1}$ and $s_{2}$ through SAfS and multiply them to $\left[\begin{array}{c}\widehat{p_{L 1}} \\ \widehat{q_{L 1}}\end{array}\right]$ and $\left[\begin{array}{l}\widehat{p_{L 2}} \\ \widehat{q_{L 2}}\end{array}\right]$, respectively, to reach to the estimated slope $\left[\begin{array}{l}p_{1} \\ q_{1}\end{array}\right]$ and $\left[\begin{array}{l}p_{2} \\ q_{2}\end{array}\right]$ along each direction:

$\left[\begin{array}{l}p_{1} \\ q_{1}\end{array}\right]=s_{1}\left[\begin{array}{c}\widehat{p_{L 1}} \\ \widehat{q_{L 1}}\end{array}\right]=\left[\begin{array}{c}s_{1} \\ 0\end{array}\right] \quad ; \quad\left[\begin{array}{l}p_{2} \\ q_{2}\end{array}\right]=s_{2}\left[\begin{array}{c}\widehat{p_{L 2}} \\ q_{L 2}\end{array}\right]=\left[\begin{array}{c}s_{2} \cos \alpha \\ s_{2} \sin \alpha\end{array}\right]$

The solution of Photometric stereo SAfS is a surface normal $\left[\begin{array}{l}p_{S A f S} \\ q_{S A f S}\end{array}\right]$ such that it arrives $\left[\begin{array}{l}p_{1} \\ q_{1}\end{array}\right]$ and $\left[\begin{array}{l}p_{2} \\ q_{2}\end{array}\right]$ simultaneously when projected to the directions denoted by $\left[\begin{array}{l}\widehat{p_{L 1}} \\ \widehat{q_{L 1}}\end{array}\right]$ and $\left[\begin{array}{c}\widehat{p_{L 2}} \\ \widehat{q_{L 2}}\end{array}\right]$ respectively. Which means $\left[\begin{array}{l}p_{S A f S} \\ q_{S A f S}\end{array}\right]$ is in fact the intersection of the perpendiculars of $\left[\begin{array}{l}\widehat{p_{L 1}} \\ \widehat{q_{L 1}}\end{array}\right]$ and $\left[\begin{array}{l}\widehat{p_{L 2}} \\ \widehat{q_{L 2}}\end{array}\right]$ originated at $\left[\begin{array}{l}p_{1} \\ q_{1}\end{array}\right]$ and $\left[\begin{array}{l}p_{2} \\ q_{2}\end{array}\right]$ :

$$
\left[\begin{array}{l}
p_{S A f S} \\
q_{S A f S}
\end{array}\right]=m_{1}\left[\begin{array}{c}
\widehat{p_{\perp 1}} \\
\widehat{q_{\perp 1}}
\end{array}\right]+\left[\begin{array}{l}
p_{1} \\
q_{1}
\end{array}\right]=m_{2}\left[\begin{array}{c}
\widehat{p_{\perp 2}} \\
q_{\perp 2}
\end{array}\right]+\left[\begin{array}{l}
p_{2} \\
q_{2}
\end{array}\right]
$$

where

$$
\begin{aligned}
& {\left[\begin{array}{c}
\widehat{p_{\perp 1}} \\
\widehat{q_{\perp 1}}
\end{array}\right]=\left[\begin{array}{cc}
0 & -1 \\
1 & 0
\end{array}\right]\left[\begin{array}{c}
\widehat{p_{L 1}} \\
\widehat{q_{L 1}}
\end{array}\right]=\left[\begin{array}{l}
0 \\
1
\end{array}\right]} \\
& {\left[\begin{array}{c}
\widehat{p_{\perp 2}} \\
\widehat{q_{\perp 2}}
\end{array}\right]=\left[\begin{array}{cc}
0 & -1 \\
1 & 0
\end{array}\right]\left[\begin{array}{c}
\widehat{p_{L 2}} \\
\widehat{q_{L 2}}
\end{array}\right]=\left[\begin{array}{c}
-\sin \alpha \\
\cos \alpha
\end{array}\right]}
\end{aligned}
$$

In equation (8), $m_{1}$ and $m_{2}$ are the two scalars to be applied on $\left[\begin{array}{l}\widehat{p_{\perp 1}} \\ \widehat{q_{\perp 1}}\end{array}\right]$ and $\left[\begin{array}{l}\widehat{p_{\perp 2}} \\ \widehat{q_{\perp 2}}\end{array}\right]$ respectively such that $\left(m_{1}\left[\begin{array}{l}\widehat{p_{\perp 1}} \\ \widehat{q_{\perp 1}}\end{array}\right]+\left[\begin{array}{l}p_{1} \\ q_{1}\end{array}\right]\right)$ and $\left(m_{2}\left[\begin{array}{c}\widehat{p_{\perp 2}} \\ \widehat{q_{\perp 2}}\end{array}\right]+\left[\begin{array}{l}p_{2} \\ q_{2}\end{array}\right]\right)$ intersects. In this case $\left(\left[\begin{array}{c}p_{S A f S} \\ q_{S A f S}\end{array}\right]=\left[\begin{array}{c}s_{1} \\ m_{1}\end{array}\right]\right)$ because the $\mathrm{p}-\mathrm{q}$ coordinate frame is always aligned to $\left[\begin{array}{l}\widehat{p_{L 1}} \\ q_{L 1}\end{array}\right]$ and hence $\left(p_{S A f S}=p_{1}=s_{1}\right)$. The problem can be simplified to only solve for $m_{1}$ that reaches magnitude $s 2$ when projected to $\left[\begin{array}{l}\widehat{p_{L 2}} \\ \widehat{q_{L 2}}\end{array}\right]$ and this can be formulated by using vector dot product:

$$
\left[\begin{array}{ll}
s_{1} & m_{1}
\end{array}\right]\left[\begin{array}{l}
\widehat{p_{L 2}} \\
\widehat{q_{L 2}}
\end{array}\right] \frac{1}{d\left(\widehat{\left.p_{L 2}, \widehat{q_{L 2}}\right)}\right.}=s_{2}
$$

where $d\left(\widehat{p_{L 2}}, \widehat{q_{L 2}}\right)$ is the length of the unit vector $\left[\begin{array}{l}\widehat{p_{L 2}} \\ \widehat{q_{L 2}}\end{array}\right]$ and equals to 1 . Hence,

$$
m_{1}=\frac{s_{2}-s_{1} \cos \alpha}{\sin \alpha}
$$

Therefore the solution of Photometric stereo SAfS $\left[\begin{array}{l}p_{S A f S} \\ q_{S A f S}\end{array}\right]$ can be expressed as:

$$
\left[\begin{array}{c}
p_{S A f S} \\
q_{S A f S}
\end{array}\right]=\left[\begin{array}{c}
s_{1} \\
\frac{s_{2}-s_{1} \cos \alpha}{\sin \alpha}
\end{array}\right]
$$

\subsection{Error propagation of Photometric Stereo SAfS}

The combined error (i.e., $\sigma_{n}^{2}$ ) of the resulted surface normal $\left[\begin{array}{l}p_{S A f S} \\ q_{S A f S}\end{array}\right]$ from SAfS can be expressed as:

$$
\sigma_{n}^{2}=\sigma_{p}^{2}+\sigma_{q}^{2}
$$

where $\quad \sigma_{p}^{2}=$ squared error of $p_{S A f S}$

$$
\sigma_{q}^{2}=\text { squared error of } q_{S A f S}
$$

From equation (11), the solution of Photometric stereo SAfS is determined by three factors: $s_{1}$ and $s_{2}$ which are solved through SAfS and reflectance modelling; and $\alpha$ which is the azimuthal difference between the two illumination directions. Hence the error of these components can be propagated to the solution by applying the Error Propagation Law to $\left[\begin{array}{l}p_{\text {SAfS }} \\ q_{\text {SAfS }}\end{array}\right]$ :

$\sigma_{p}^{2}=\left(\frac{\partial p_{S A f S}}{\partial s_{1}}\right)^{2} \sigma_{S 1}^{2}+\left(\frac{\partial p_{S A f S}}{\partial s_{2}}\right)^{2} \sigma_{S 2}^{2}+\left(\frac{\partial p_{S A f S}}{\partial \alpha}\right)^{2} \sigma_{\alpha}^{2}$

$\sigma_{q}^{2}=\left(\frac{\partial q_{S A f S}}{\partial s_{1}}\right)^{2} \sigma_{s 1}^{2}+\left(\frac{\partial q_{S A f S}}{\partial s_{2}}\right)^{2} \sigma_{S 2}^{2}+\left(\frac{\partial q_{S A f S}}{\partial \alpha}\right)^{2} \sigma_{\alpha}^{2}$

where $\begin{array}{lll}\frac{\partial p_{S A f S}}{\partial s_{1}}=1 & ; & \frac{\partial p_{S A f S}}{\partial s_{2}}=0 \\ \frac{\partial q_{S A f S}}{\partial s_{1}}=-\frac{1}{t_{n}} & ; & \frac{\partial q_{S A f S}}{\partial S_{2}}=1\end{array}$

$$
\frac{\partial q_{\text {SAfS }}}{\partial s_{1}}=-\frac{1}{\tan \alpha} ; \quad ; \quad \frac{\partial q_{\text {SAfS }}}{\partial s_{2}}=\frac{1}{\sin \alpha}
$$

Assuming there is no or neglectable error from $\alpha$ (i.e., $\sigma_{\alpha}^{2}=0$ ) and combing equation (13) and (14) to (12), the final error term is then:

$$
\sigma_{n}^{2}=\sigma_{p}^{2}+\sigma_{q}^{2}=\left(1+\frac{1}{\tan ^{2} \alpha}\right) \sigma_{s 1}^{2}+\left(\frac{1}{\sin ^{2} \alpha}\right) \sigma_{s 2}^{2}
$$

Note that equation (15) describes the effects of errors in $s_{1}$ and $s_{2}$ to the final solution of surface normal $\left[\begin{array}{l}p_{\text {SAfS }} \\ q_{S A f S}\end{array}\right]$. Errors related to SAfS algorithms such as reflectance modelling error and albedo error are represented by $\sigma_{s 1}^{2}$ and $\sigma_{s 2}^{2}$ and therefore not explicitly presented in equation (15). To explicitly evaluate the effects of each of the SAfS error components (e.g., Albedo error, modelling error) one has to perform error propagation on $s_{1}$ and $s_{2}$ and propagate them to equation (15).

Equation (15) presents an interesting relationship between the illumination azimuthal difference of the images (i.e., $\alpha$ ) and the final error term $\sigma_{n}^{2}$ : The coefficient of $\sigma_{s 1}^{2}$ behaves in a quadratic fashion and reaches minimal when $\left(\alpha=90^{\circ}\right)$ where $\left(\tan ^{2} \alpha=+\operatorname{In} f\right)$ and hence $\frac{1}{\tan ^{2} \alpha}$ is practically zero. On the other hand, it approaches maximal when $\left(\alpha=0^{\circ}\right.$ or $\left.180^{\circ}\right)$ 
where $\left(\tan ^{2} \alpha=0\right)$ and hence $\left(\frac{1}{\tan ^{2} \alpha}=+\operatorname{Inf}\right)$. The coefficient of $\sigma_{s 2}^{2}$ behaves in similar pattern where $\frac{1}{\sin ^{2} \alpha}$ reaches minimal when $\left(\alpha=90^{\circ}\right)$ and maximal when $\left(\alpha=0^{\circ}\right.$ or $\left.180^{\circ}\right)$.

Considering both terms, $\sigma_{n}^{2}$ approaches minimal when ( $\alpha=$ $90^{\circ}$ ), which implies photometric stereo pair (i.e., two images) with azimuthal difference $\alpha$ close to $90^{\circ}$ is likely to produce better SAfS results and the performance decreases as $\alpha$ deviates from $90^{\circ}$. One reasoning for such behaviour is that at $90^{\circ}$ both directions are independent from each other and therefore $\sigma_{s 1}^{2}$ will only affect $p_{S A f S}$ and $\sigma_{p}^{2}$ while $\sigma_{S 2}^{2}$ will only affect $q_{S A f S}$ and $\sigma_{q}^{2}$; while in other cases $\sigma_{S 1}^{2}$ will affect both $\left[\begin{array}{l}p_{S A f S} \\ q_{S A f S}\end{array}\right]$ as well as $\left[\begin{array}{l}\sigma_{p}^{2} \\ \sigma_{q}^{2}\end{array}\right]$ and its effect increases as the two directions get closer to be collinear. At $0^{\circ}$ or $180^{\circ}$, the two illumination directions become collinear (coplanar in $3 \mathrm{D}$ space) and therefore it is insufficient to obtain $\left[\begin{array}{l}p_{S A f S} \\ q_{S A f S}\end{array}\right]$ accurately without external constraints.

\section{EXPERIMENTAL ANALYSIS}

\subsection{Validation procedure}

In order to verify the error propagation model described in section 3, real lunar images and reference DEM data were used and the results were analysed. The validation includes two parts. One is a check of the surface normals which is directly related to the previously described theoretical analysis. The other is a comparison on the final generated DEMs.

The general routine for the validation is as follows: (1) Generate SAfS DEM for each photometric stereo pair; (2) Resample the SAfS DEMs so that they synchronized with the reference DEM; (3) Shift vertically the SAfS DEMs by aligning their mid-point heights to that of the of reference DEM; (4) Calculate angular and vertical error.

Angular error refers to the angle between the surface normal on SAfS DEM and that on the reference DEM. It is calculated by using vector dot product and evaluated at each point $(x, y)$ :

where

$$
e_{x, y}^{a n g}=\left.\cos ^{-1}\left(\frac{n_{S A f S}^{T} n_{\text {ref }}}{d\left(n_{S A f S}\right) d\left(n_{r e f}\right)}\right)\right|_{x, y}
$$

$$
\begin{aligned}
& e_{x, y}^{a n g}=\text { angular error at point }(x, y) \\
& n_{S A f S}=\left[\begin{array}{c}
\frac{\partial Z_{\text {SAfS }}}{\partial x} \\
\frac{\partial Z_{\text {SAfS }}}{\partial y} \\
1
\end{array}\right] \quad ; \quad n_{\text {ref }}=\left[\begin{array}{c}
p_{\text {ref }} \\
q_{\text {ref }} \\
1
\end{array}\right] \\
& d(n)=\sqrt{n^{T} n}
\end{aligned}
$$

In addition to the angular analysis which is more directly correlated to the error propagation in section 3, vertical error analysis is also conducted and analysed for a more comprehensive evaluation. Vertical error refers to the height difference between the SAfS DEM and the reference DEM in absolute value and is evaluated at each point $(x, y)$ :

$$
e_{x, y}^{v e r t}=\left|Z_{S A f S}-Z_{r e f}\right|_{x, y}
$$

where

$$
\begin{aligned}
& e_{x, y}^{\text {vert }}=\text { vertical error at point }(x, y) \\
& Z_{\text {SAfS }}=\text { height of SAfS DEM at point }(x, y) \\
& Z_{\text {ref }}=\text { height of reference DEM at point }(x, y)
\end{aligned}
$$

The quality of a photometric stereo pair is described by the mean and maximum errors of all points within the domain. The albedo is also set to constant for all pairs so that the error brought by albedo will be consistent for all set.

\subsection{Dataset}

Four Calibrated Data Record (CDR) images from the Lunar Reconnaissance Orbiter Camera - Narrow Angle Camera (LROC NAC) were utilized and tested (Figure 2). The image set produces 6 photometric stereo pairs with different illumination azimuthal difference (Table 3). The images were co-registered and resampled to $1 \mathrm{~m} /$ pixel so that the pixels are aligned and synchronized. The dimension of the co-registered images is $1024 \times 6144$ pixels which is about $6 \mathrm{~km}^{2}$ large. They are then geo-referenced to a reference DEM of the same area created independently using photogrammetric methods. Both the images and the DEM are available in the LROC archive (http://lroc.sese.asu.edu/archive). The illumination azimuthal difference of the photometric stereo pairs ranges from $27^{\circ}$ to $154^{\circ}$ with a pair close to $90^{\circ}$. It covers the key angles needed to present the quadratic behaviour, if any, as described in section 3.
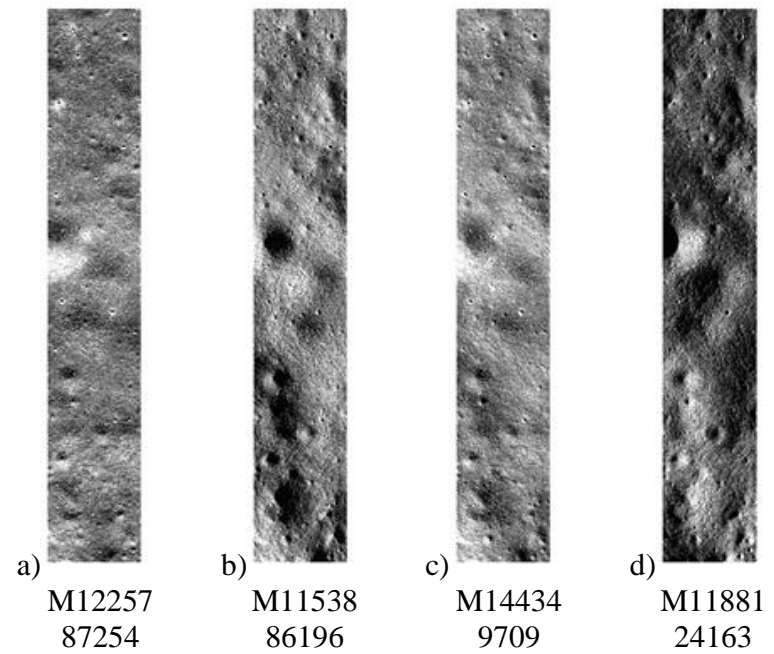

Figure 2. Co-registered LROC NAC CDR images for experimental analysis

\begin{tabular}{|c|c|c|c|c|}
\hline Image ID & $\begin{array}{c}\text { M12257 } \\
87254\end{array}$ & $\begin{array}{c}\text { M11538 } \\
86196\end{array}$ & $\begin{array}{c}\text { M14434 } \\
9709\end{array}$ & $\begin{array}{c}\text { M11881 } \\
24163\end{array}$ \\
\hline $\begin{array}{c}\text { M12257 } \\
87254\end{array}$ & & 68.79 & 41.82 & 84.92 \\
\hline $\begin{array}{c}\text { M11538 } \\
86196\end{array}$ & & & 26.97 & 153.72 \\
\hline $\begin{array}{c}\text { M14434 } \\
9709\end{array}$ & & & & 126.75 \\
\hline $\begin{array}{c}\text { M11881 } \\
24163\end{array}$ & & & & \\
\hline
\end{tabular}

Table 3. Illumination azimuthal difference of image pairs in degrees 


\subsection{Experimental results}

\subsubsection{Angular error analysis}

Figure 4 presents the angular error DEMs calculated by equation (16), no visually apparent difference between these DEMs can be detected except for Figure $4 \mathrm{~d}$, e and $\mathrm{f}$ where a darker shade exist in middle-left of the area while the others do not. This is believed to stem from the shadows of the larger crater in one of those member images. The error statistics of the dataset are calculated and plotted with respect to illumination azimuthal difference in Figure 5: the maximum angular error curves (Figure 5a) generally present a U-shape pattern with around $90^{\circ}$ as minimal. Although the mean error curve (Figure 5b) also shows a slight U-shape pattern, they are not as apparent as the maximum curve and there is a drop at $26.97^{\circ}$ which does not perfectly follow the error propagation model previously described.

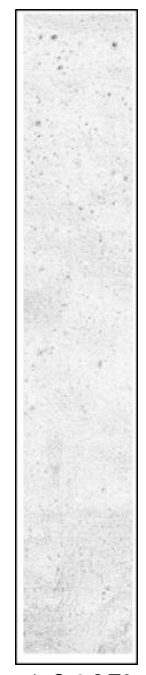

a) $26.97^{\circ}$

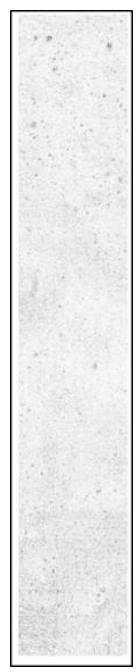

d) $84.92^{\circ}$

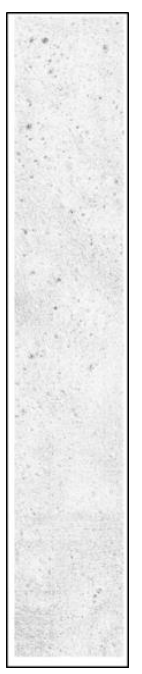

b) $41.82^{\circ}$

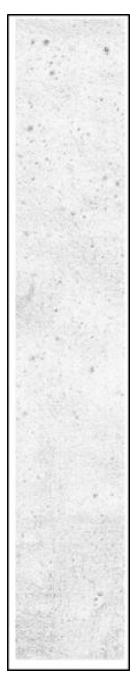

e) $126.75^{\circ}$
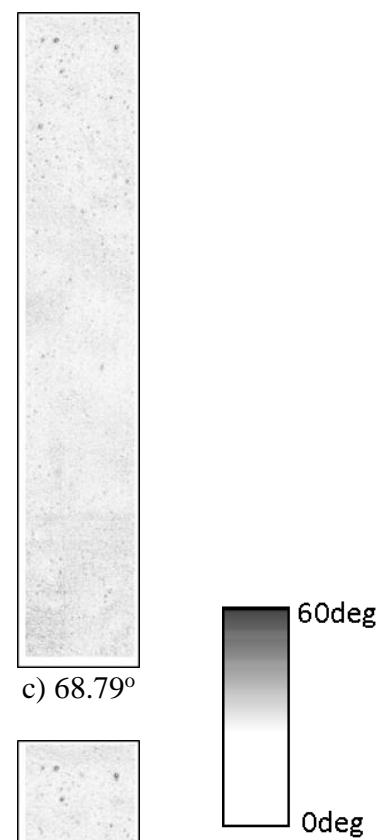

Colour scale $\left({ }^{\circ}\right)$ c) $68.79^{\circ}$
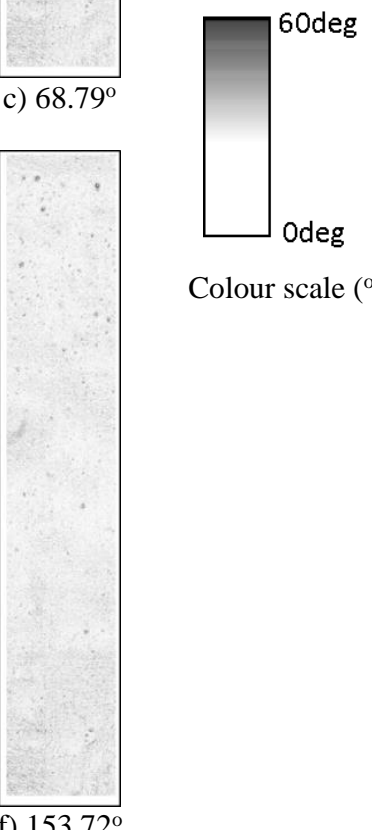

Figure 4. Angular difference DEMs ( $1 \mathrm{~m} /$ pixel) at each illumination azimuthal difference

We look into the issue by computing the angular residuals between the surface normals derived from SAfS DEMs and their corresponding estimated surface normal. This is computed by substituting $n_{\text {ref }}$ by $\left[\begin{array}{llll}p_{S A f S} & q_{S A f S} & 1\end{array}\right]^{T}$ in equation (16).
This is to address the correspondence between the SAfS DEM and the estimated surface normal field and found that the correspondence decreases as the illumination directional difference deviates from $90^{\circ}$. This means that one possibility for such exception is the uncertainties between the final DEM and the estimated surface normal field. These uncertainties decrease the DEM's correspondence and, with accumulated errors in other factors such as albedo, reflectance modelling and noises, may lead to unstable analysis findings. Extra datasets and future investigations are required in order to make conclusive remarks. One interesting finding from Figure 5 is that the errors of angular difference larger than $90^{\circ}$ tend to rise at a slower rate than those smaller than $90^{\circ}$, creating an unbalanced quadratic curve. This may suggest if $90^{\circ}$ is not available, photometric stereo pairs with angles larger than $90^{\circ}$ might yields better results than those smaller than $90^{\circ}$. This pattern also indicates a possibility of a more complexed behaviour of error propagation based on the model derived in section 3 .

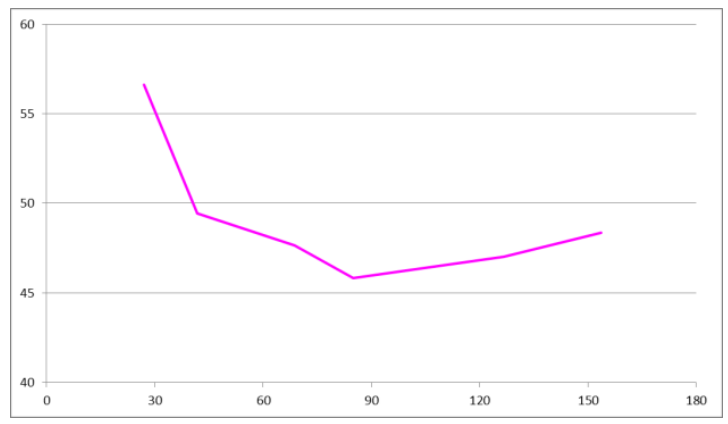

a) Maximum angular error (vertical axis: $40^{\circ}-60^{\circ}$ )

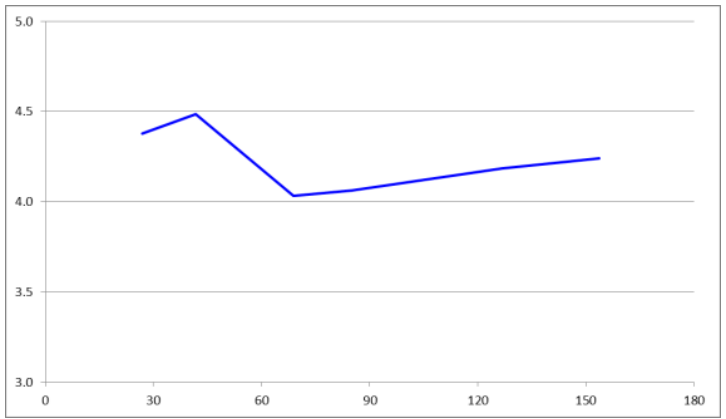

b) Mean angular error (vertical axis: $3^{\circ}-5^{\circ}$ )

Figure 5. Angular error statistics. Horizontal axis: illumination azimuthal difference $0^{\circ}-180^{\circ}$.

\subsubsection{Vertical error analysis:}

Figure 6 provides a 3D visual comparison for each SAfS DEM. While almost all of them cannot reach to the highest elevation presented in the reference DEM (Figure 6g), they largely preserve the details. Some small differences can be noted in the upper part of the DEMs where some of the DEMs present a smooth terrain (Figure 6b, c and d) while others are rugged with their upper edges slightly lifted up (Figure 6a, e and f). The vertical difference DEMs in Figure 7 present the error between the SAfS DEM and reference DEM calculated by equation (17). Apparent vertical error is noted at the bottom side of the area where the SAfS DEMs were lower than the reference DEM. The DEMs in Figure 7d, e and f present similar pattern, this is because one image was shared among these pairs. Notably the photometric stereo pair at $41.82^{\circ}$ (Figure $7 b$ ) contains more dark 
pixels than the others, especially around the edges of the middle and upper areas. This corresponds to the peak value in Figure $8 \mathrm{~b}$.

The vertical error statistics presented in Figure 8 generally present similar patterns aforementioned. However there is a drastic drop in mean vertical error (Figure $8 \mathrm{~b}$ ) at $26.97^{\circ}$ which becomes the minimal of the curve. It is believed that similar reasons previously described (section 4.3.1) such as correspondence errors and other accumulated random and systematic errors were taking effect. This will also need future investigations to make conclusions.

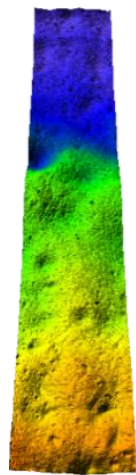

a) $26.97^{\circ}$

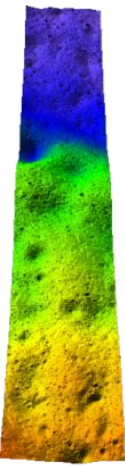

d) $84.92^{\circ}$

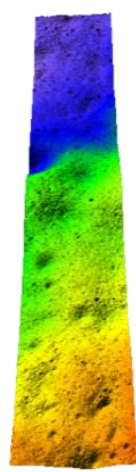

b) $41.82^{\circ}$

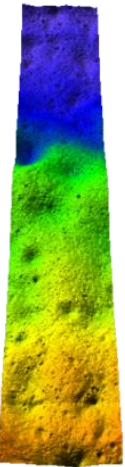

e) $126.75^{\circ}$

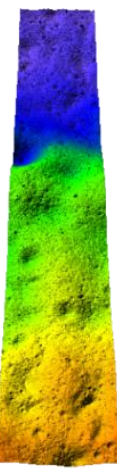

c) $68.79^{\circ}$

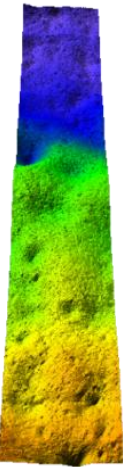

f) $153.72^{\circ}$

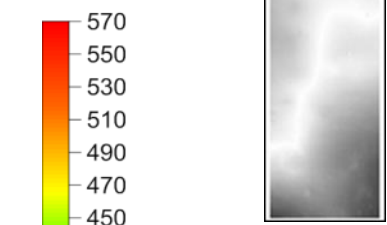

d) $84.92^{\circ}$

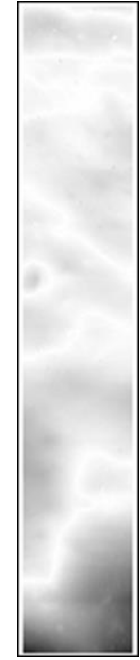

e) $126.75^{\circ}$

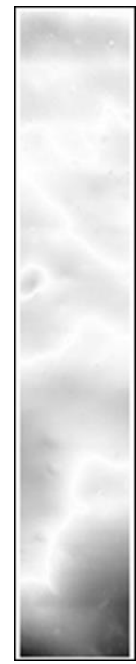

f) $153.72^{\circ}$
Figure 7. Vertical difference DEMs (1m/pixel) at each illumination azimuthal difference

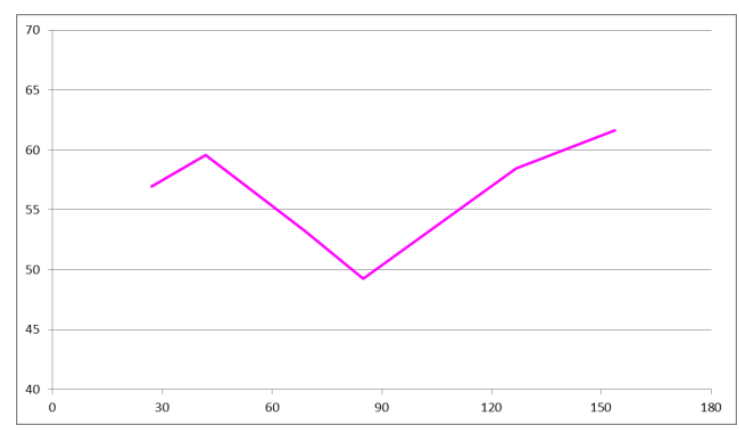

a) Maximum vertical error (vertical axis: $40 \mathrm{~m}-70 \mathrm{~m}$ )

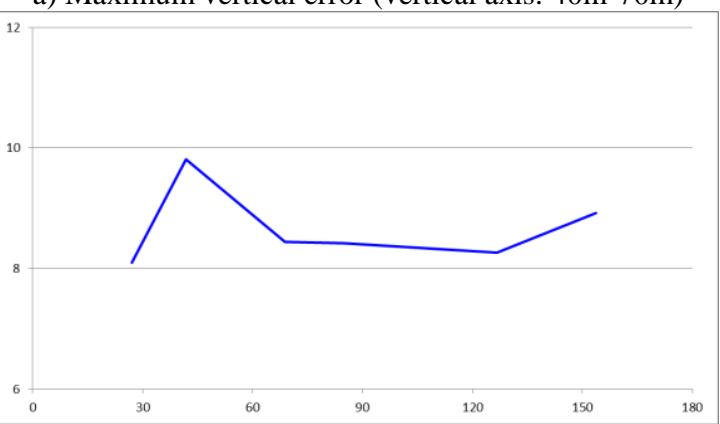

b) Mean vertical error (vertical axis: $6 \mathrm{~m}-12 \mathrm{~m}$ )

Figure 8. Vertical error statistics. Horizontal axis: illumination azimuthal difference $0^{\circ}-180^{\circ}$.

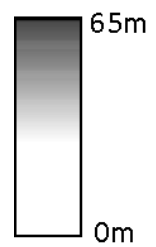

Colour scale

(m)

\section{CONCLUSION AND DISCUSSION}

This paper presents a straight forward method in lunar surface reconstruction through photometric stereo SAfS. The results indicate plausible surface recovery with virtually no extra information for initial estimation. Error propagation of single photometric stereo pair SAfS performance with respect to the horizontal angular difference between two illumination directions is developed and suggests better performance when the angular difference approaches $90^{\circ}$. Experimental analysis using real LROC NAC dataset generally follows this pattern with considerable uncertainties. The actual error propagation behaviour of photometric stereo SAfS may be more complicated than what have been derived here. However, it is believed that 
the error propagation model described here provides the basic structure for future derivation of more sophisticated models.

\section{ACKNOWLEDGEMENTS}

The work was supported by a grant from the Research Grants Council of Hong Kong (Project No: PolyU 152086/15E) and a grant from the National Natural Science Foundation of China (Project No: 41471345).

\section{REFERENCES}

Capanna, C., Gesquière, G., Jorda, L., Lamy, P., Vibert, D., 2013. Three-dimensional reconstruction using multiresolution photoclinometry by deformation. The Visual Computer, 29(6-8), pp. 825-835.

Frankot, R. T., Chellappa, R., 1988. A Method for Enforcing Integrability in Shape from Shading Algorithms. IEEE Transactions on Pattern Analysis and Machine Intelligence, 10(4), pp. 439-451.

Grumpe, A., Belkhir, F., Wöhler, C., 2014. Construction of lunar DEMs based on reflectance modelling. Advances in Space Research, 53(12), pp. 1735-1767.

Horn, B. K. P., 1977. Understanding Image Intensities. Artificial Intelligence, 8(2), pp. 201-231.

Horn, B. K. P., 1990. Height and Gradient from Shading. International Journal of Computer Vision, 5(1), pp. 37-75.

Hapke, B., 2002. Bidirectional Reflectance Spectroscopy. 5. The Coherent Backscatter Opposition Effect and Anisotropic Scattering. Icarus, 157(2002), pp. 523-534.

Lohse, V., Heipke, C., 2004. Multi-image Shape-from-shading: Derivation of Planetary Digital Terrain Models Using Clementine Images. The International Archives of the Photogrammetry, 35(B4), pp. 828-833.

McEwen, A. S., 1991. Photometric Functions for Photoclinometry and Other Applications. Icarus, 92(1991), pp. 298-311.

Woodham, R. J., 1980. Photometric method for determining surface orientation from multiple images. Optical Engineering, 19(1), pp. 139-144.

Wu, B., Liu, W.C., Grumpe, A., Wöhler, C., 2017. Construction of Pixel-Level Resolution DEMs from Monocular Images by Shape and Albedo from Shading Constrained with LowResolution DEM. ISPRS Journal of Photogrammetry and Remote Sensing, doi: 10.1016/j.isprsjprs.2017.03.007. 